\title{
SELF-CALIBRATION OF ULTRASONIC TRANSDUCERS IN AN INTELLIGENT DATA ACQUISITION SYSTEM
}

\author{
Chris Papageorgiou, Theodore Laopoulos
}

\author{
Electronics Lab. Physics Dept., Aristotle University of Thessaloniki, Thessaloniki, 54124, Greece, e-mail: \\ papageorgiou@physics.auth.gr.
}

\begin{abstract}
The rapid growth of powerful single-chip microcomputers for monitoring and data acquisition applications permits nowadays the design of advanced measuring systems. The work reported here is presenting an advanced online monitoring configuration in order to improve the performance and extend the lifetime of ultrasonic transducers by applying an automated testing and calibration technique. The operation of this instrumentation system is based on the fast measurement of frequency and amplitude, performed by the proposed configuration. The combination of this information with the time of flight of each pulse-train is then used to derive practically all characteristics of ultrasonic transducers. Due to its low cost and small size, the system can be used either for characterization and classification of transducers, or as a self-testing and automated calibration section within any high performance ultrasonic system.
\end{abstract}

Keywords: - Ultrasonic transducers, transducer calibration, transducer characterization

\section{INTRODUCTION}

The use and the interest in air coupled ultrasonic transducers have increased in recent years. Ultrasonic transducers are widely used in a variety of applications and in technological areas with significant differences in performance characteristics, operating environment, specifications, etc. Ultrasonic instrumentation and measurement systems can be found in applications varying from underwater or industrial systems to medical imaging, nondestructive evaluation, and robotics. Piezoelectric transducers cannot be economically produced to generate either a controlled radiation pattern or a reproducible amplitude and frequency response. Transducer sensitivities and resonant frequencies tend to change with time and usage with an average life expectancy, which is sometimes limited to a few months of continuous use.

With the advances made in air transducer technology, there is a need for suitable calibration tools for characterization of airborne transducers. The increased usage of the transducers, results to an enhanced need for precise knowledge of the characteristics of the transducers and consequently for new precise methods to test and evaluate their performance. The more well known the operating characteristics of the transducers are, the more accurate the signal analysis will be. These characteristics must be verified for each transducer before installing it to a high performance system, and furthermore there must be established a test routine to check periodically transducers' operation. It is not surprising to find transducers that do not meet expectations or others that suffer from severe performance degradation (depending on the operating environment). Various methods have been proposed for measuring the performance characteristics of ultrasonic transducers, nearly all of them based on complicated systems from both; the hardware and the software point of view $[1,2,3,6]$. Unix workstations and spectrum analyzers are used by a number of teams together with certain mathematical calculations and advanced techniques like neural configurations and pattern recognition [1]. The aim is to identify and classify automatically ultrasonic transducers according to the value of certain characteristics of the transducers [1] or the echo signal [7].

Yet, there are two weak points to this approach; (1) the tests cannot be performed on site, as the system is operating and (2) a complicated and expensive test configuration is needed to perform the measurements and to analyze the data. Further more not all of the measured characteristics are really needed for a proper evaluation of the transducer operation. In most cases, once a certain type of ultrasonic transducer is chosen at the design and development phase building a prototype system, then only a small number of characteristics need to be measured for the evaluation routine. These 
characteristics are basically the level of the transmitted/received signal (sensitivity) and the resonance (peak) frequency. But even in cases where a more detailed analysis of the transducer is needed, nearly all information can be retrieved from measurements of the amplitude of the received signal (sensitivity of the transmitter receiver pair) versus frequency and distance.

This work presents a different approach based on the capabilities of modern DSP microcontrollers. The method implemented by this system is based on its capability of performing fast measurements of these characteristics. The proposed microcontroller configuration (shown in fig. 1) is measuring the frequency and the amplitude of each period of the incoming signal, along with the time of arrival of each period. These measured values are then used to provide the desired characterization of the transducer. A description of this automated test configuration is given in the following, along some illustrative examples of the type of measurements that can be performed.

\section{SYSTEM OPERATION}

The characteristics of an automated system for the evaluation of ultrasonic transducers are presented in this section. The system is basically intended for evaluation of transducers operating in the range of $40 \mathrm{kHz}$, but it may also be used for higher frequency transducers, with limited accuracy or response time, due to the operating frequency (clock) of the digital circuitry. This instrumentation configuration is designed with the aim to perform two main operations:

- Automated testing of the operating condition of ultrasonic transducers (compliance to certain characteristics), and

- Measurement of the values of important parameters of these transducers so that an automated characterization and calibration procedure may be applied

The basic features of such a system includes the measurement of only two characteristic quantities of the received signal; amplitude and frequency. Yet these features must be accompanied by other very important capabilities. These capabilities of the testing configuration which provide the necessary conditions for the successful attainment of the task are:

1. precise control of the characteristics of the transmitted pulse train in terms of frequency, amplitude and number of pulses.

2. fast measurement of the frequency value and of the amplitude value, which should be performed for each period of the received signal (measurement resolution of one single period of the received signal).

3. precise recording of the arrival time for each period of the received signal.

4. capability to perform mathematical calculations in order to derive the values of the desirable quantities from the measured data.

5. minimum hardware requirements in order to include the overall testing configuration into any high performance instrumentation system which is using the ultrasonic transducers under test.

So the basic operation of the system is the accurate

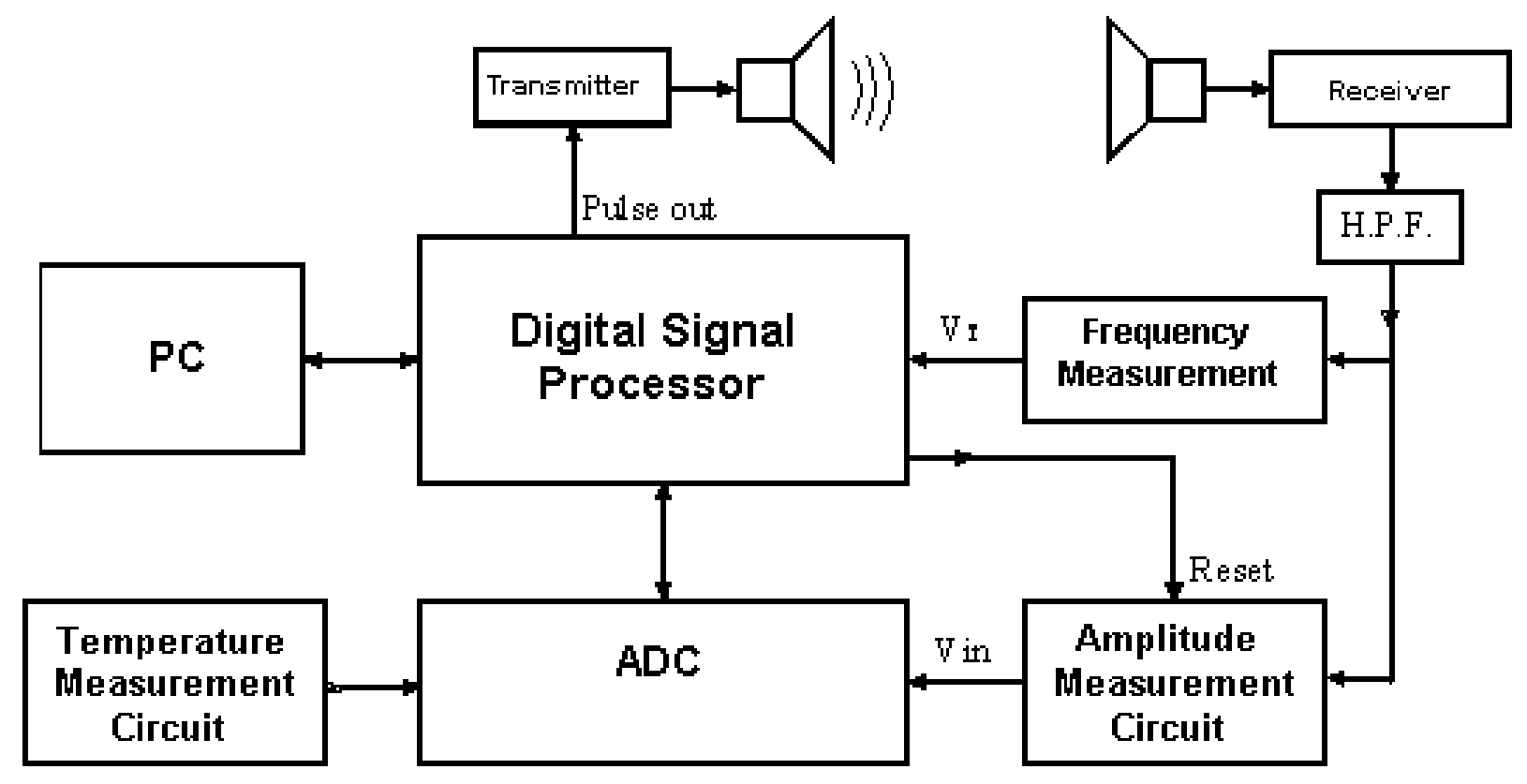

Fig. 1 - Block diagram of the proposed system. 
measurement of the amplitude and frequency values of each consecutive period of the input signal. The measurement system provides therefore at the output three types of information about the following:

1. Steady state response (received amplitude for a constant frequency signal) in order to generate from a number of measurements, a diagram of sensitivity versus frequency.

2. Steady state response (amplitude vs frequency) with the additional capability of analyzing the measured values in order to provide at the output additional characteristics about each one of the transducers of the pair. This feature is performed by a novel software which is developed especially for this case. Further information about this procedure are given in the next paragraph.

3. Transient response for each application (the amplitude variation over time for constant frequency of the transmitted signal). This parameter (short of time constant for the overall ultrasonic system) is very useful in cases of instrumentation configurations operating in non-uniform environments (i.e. ultrasonic analysis of liquids) or for systems operating at high repetition rates.

The proposed configuration is also capable of performing scheduled measurements of the parameters of the transducers in high performance Ultrasonic measuring instruments for calibration purposes, and storing the data locally in a memory module. For example the system automatically adjusts the desired gain and resonant frequency during the calibration phase to compensate for transducer variations by means of a DSP microcontroller smart routine. Such systems are used for accurate distance measurements [4], or remote vibration measurements [6]. The calibration data may then be transmitted to a service data base system for further analysis either by using a cellular phone network or via a UHF transceiver. Validation of measurements is achieved by the system's self check control and warning signals can be sent to authorized personnel in case of errors as out of range operating frequencies and amplitude gains.

The proposed practical configuration of such an automated system is shown in fig. 1, which has been developed around a powerful microcontroller with digital signal processing capabilities. The DSP56002 microcontroller, which is the core unit of this configuration, is a 24bits, $40 \mathrm{MHz}$ single-chip computer with internal RAM, and special timing measurement technique for high accuracy measurements of time intervals (period or frequency). The microcontroller is capable of driving the ultrasonic transmitter with a precisely determined pulse train. This means that the frequency (or actually the period) of each consecutive pulse sent to the transmitter can be controlled, along with the total number of the pulses and the amplitude of the pulse train.

At the other end, an analog interfacing circuit is employed to provide the same microcontroller with the appropriate input information (about the received ultrasonic signal). At the input, right after the ultrasonic receiver there is an analog signal conditioning section, which is formed by a controlled amplifier and a high pass filter (used to reduce possible low-frequency interference). Then the input signal enters two different paths: a frequency measurement path with a specially designed high-speed comparator, and an amplitude measurement path with a maximum value circuit. From the first path the microcontroller gets the information about the duration of each consecutive period, and from the second the digital data corresponding to the value of the amplitude for this period.

The first circuit is a high-speed comparator, which triggers the timing input of the microcontroller at the exact time instants of the beginning of each period of the received signal. The timing measurement technique employed by the DSP56002 is based on a free running 24bits counter. The trigger signal from the comparator actually generates an interrupt, which loads the content of the free-running counter to a 24 bit timer count register. User's program then can read this register, subtract consecutive values and therefore calculate the time interval between two events (the time from the beginning of one period till the beginning of the next one, etc). The frequency value may then be calculated from the value of the period with an accuracy depended on the clock frequency of the timer .

On the second path, a precision half wave rectifier followed by a Sample-and-Hold circuit drives the A/D converter with the maximum value of each period (that is the amplitude value of each period). The accuracy of the amplitude measurement is mainly depended on the $\mathrm{A} / \mathrm{D}$ converter, which is a 10bits parallel output converter with 900nsecs max conversion time and $800 \mathrm{KHz}$ sampling rate. The final figures for the accuracy of the measurements of the frequency and the amplitude of each period of the incoming signal are $25 \mathrm{nsecs}$ for the period and $5 \mathrm{mV}$ (or $1 / 1000$ ) for the amplitude.

\section{ANALYSIS OF MEASUREMENTS}

A major goal of this research work was to provide solutions for the practical problems arising with the use of low cost ultrasonic transducers in measurement systems.

As was mentioned previously a significant problem in such cases is related to the exact value of 
the operating frequency and the shape (or width) of the frequency response curve for each transducer. It is important to note that if such transducers are to be used in a rather conventional way, then the effect of the limited knowledge about the value of the above parameters is not significant and may be neglected in most cases. Yet, as the transducers are operated in a more sophisticated way (i.e. frequency modulation, time response measurements, etc), then the precise knowledge of these parameters becomes more and more important. Most of the recent publications about ultrasonic measurement techniques (from this and from other teams as well) involve such dynamic type of measurements with the ultrasonic transducers in order to increase accuracy and efficiency. It is therefore a rather important point of the design process to know the exact values of the main characteristics of the transducers used in a system.

Our research work in this direction was strongly related to the possibility of having all this information available throughout the operational life of the instrument. That is to measure periodically the corresponding values and then to be able of readjusting the system in case of strong deviations of the transducers characteristics. For this reason we have moved in the direction of using the automated measurement capabilities in order to provide the information about the behavior of transducers. The main limitation of this approach is that one is measuring the ultrasonic system as a whole and is not able to identify the sending transducer from the receiving one. Yet, this information is of minor practical importance since the two transducers are operating as a pair in the system and one is not really interested if for example a change in the bandwidth value is in the transmitting or the receiving side: the result on the system is the same since in both cases the overall system bandwidth is affected.

These four characteristic parameters of the transducer pair (frequency of the maximum response and bandwidth for each one of the transducers) are considered as the only main parameters of interest since the well-known second order model is adopted for the transducers. The calculation procedure is determined on the basis of combining the information from the experimental measurements of the transmitter- receiver pair with their theoretical fourth order model. This process of analyzing the measurements can certainly be automated and may be implemented by an appropriate algorithm on a trial and error basis of estimation of the optimum values for these four parameters. The software that has been developed calculates the values of the four parameters: $\mathrm{f}_{0 \mathrm{~T}}, \mathrm{BW}_{\mathrm{T}}, \mathrm{f}_{0 \mathrm{R}}, \mathrm{BW}_{\mathrm{R}}$, with the use of a specific criterion: the theoretical curve $\mathrm{H}(\mathrm{s})$ generated from the model of the transducers pair, is to approach as much as possible to the experimental data. The experimental data of the frequency response of the pair of the transducers are actually a set of points in the form of $\left(f_{i}, a_{i}\right)$ with $f_{i}$ the frequency and $a_{i}$ the norm of $\mathrm{H}(\mathrm{s})$, namely the ratio of the amplitude of the output to the amplitude of the input.

The program sweeps a range of values for each one of the parameters $\mathrm{f}_{0 \mathrm{~T}}, \mathrm{BW}_{\mathrm{T}}, \mathrm{f}_{0 \mathrm{R}}, \mathrm{BW}_{\mathrm{R}}$, calculates the theoretical curve for each cae and compares the curve to the experimental data. Actually this comparison is performed by means of a mathematical criterion developed for the implementation of this procedure. The final output is the most optimal set of the possible values of the four parameters that fit to the experimental data.

The criterion of most optimal approach is the minimization of the sum:

$$
\sigma=\sum_{i}^{N}\left|1-\frac{\frac{|H(f i)|^{2}}{a_{i}{ }^{2}}}{\frac{1}{N} \sum_{i}^{N} \frac{|H(f i)|^{2}}{a_{i}{ }^{2}}}\right| a_{i}{ }^{2}
$$

This criterion tries to approach in shape the theoretical curve $|\mathrm{H}(\mathrm{s})|^{2}$ in the experimental points $a_{i}^{2}$, giving more weight in the points with large $a_{i}^{2}$ and least weight in the points with small $\mathrm{a}_{\mathrm{i}}^{2}$.

The objective of this procedure is to evaluate how close the theoretical analysis to experimental behavior is by estimating the approximation of experimental data with a continuous function. The expression of this function is taken for granted (based on theoretical analysis) and is the expression of the transfer function of the system transmitterreceiver:

$$
H(s)=k \frac{s}{s^{2}+2 \pi B W_{T} s+\left(2 \pi f_{0 T}\right)^{2}} \frac{s}{s^{2}+2 \pi B W_{R} s+\left(2 \pi f_{0 R}\right)^{2}}
$$

For a successful approximation this routine searches for the best values of the four parameters which appear in the above expression and are: $\mathrm{f}_{0 \mathrm{~T}}$ (resonance frequency of the transmitter), $\mathrm{BW}_{\mathrm{T}}$ (bandwidth of the transmitter), $\mathrm{f}_{0 \mathrm{R}}$ (resonance frequency of the receiver), and $\mathrm{BW}_{\mathrm{R}}$ (bandwidth of the receiver).

These best values which are the results of the software routine give the best approximation, according to the approximation criterion used for their selection. 


\section{EXPERIMENTAL RESULTS}

The proposed system as shown in fig. 1 has been implemented and used to evaluate pairs of ultrasonic transducers working in the range of $40 \mathrm{kHz}$. Some illustrative results of a number of pairs and for different operating conditions are presented in figs 2 , 3 , and 4 . The system has the capability of measuring the sensitivity curve of different pairs (fig. 2). The curves shown in fig. 2 represent the frequency response diagram for two different pairs of transducers. The quantity a of the y-axis is the ratio of the received amplitude to the transmitted one, representing thus the sensitivity of the transmitter receiver pair. The same quantity is also used in the transient response diagram (fig. 3). The resonance frequency value can be derived from the curves of fig. 2, even in cases where the transmitter and the receiver have different characteristics (fig.2, curve 2).

A second type of measurements is shown in fig. 3 where two different pairs of transducers are tested for the same operating frequency.

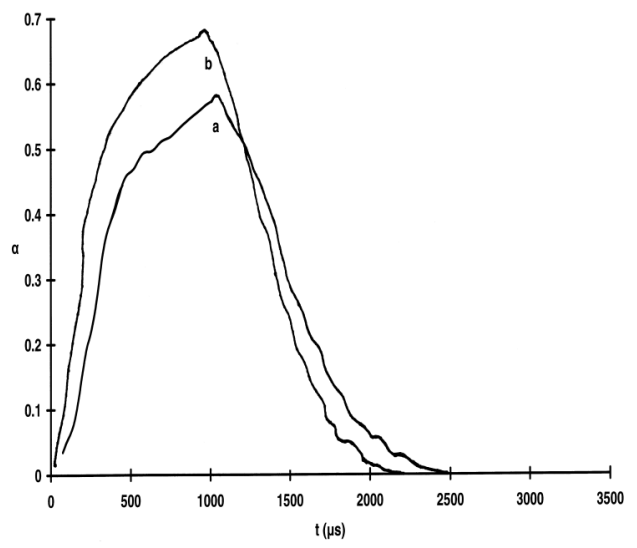

Fig. 3 -Transient response of two different pairs of transducers triggered with the same number of transmitted pulses.

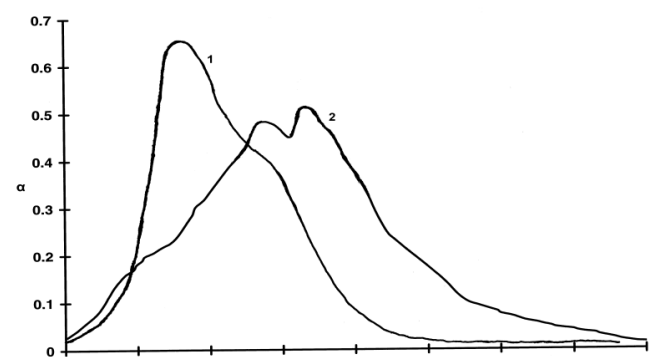

Fig. 2 - Frequency response of two different

Clearly the kind and the quality of the transducer is affecting the level and the shape of the transient response (sensitivity over time). This figure also presents a comparison of the transient response for these pairs and with the same number of transmitted pulses. Measurements of this kind can be used to define the minimum number of transmitted pulses for optimum response or the sensitivity level for a certain number of pulses. It is shown for example that for the case shown here a transmitted burst of 30 pulses gives different response for each pair and depends on the kind, the quality and ageing of the crystal.

Figures 4 and 5 show the amplitude response of a coupled pair of transducers. Dotted curves represent experimental data, while continuous curves represent theoretical data. For this pair the amplitude response was measured as a function of frequency.

The transmitting signal was a sweep ultrasonic signal from $37 \mathrm{KHz}$ to $45 \mathrm{KHz}$, with a steep of $100 \mathrm{~Hz}$ and steep duration of 100 periods. Afterwards these sets of values (amplitude, frequency) are imported in the analysis software which has been described in the previous paragraph. Once the results of this analysis succeed to provide a theoretical curve (continuous line) which closely follows the experimental measurements (dots), then the characteristic quantities of each one of the transducers is obtained (center frequency and bandwidth).

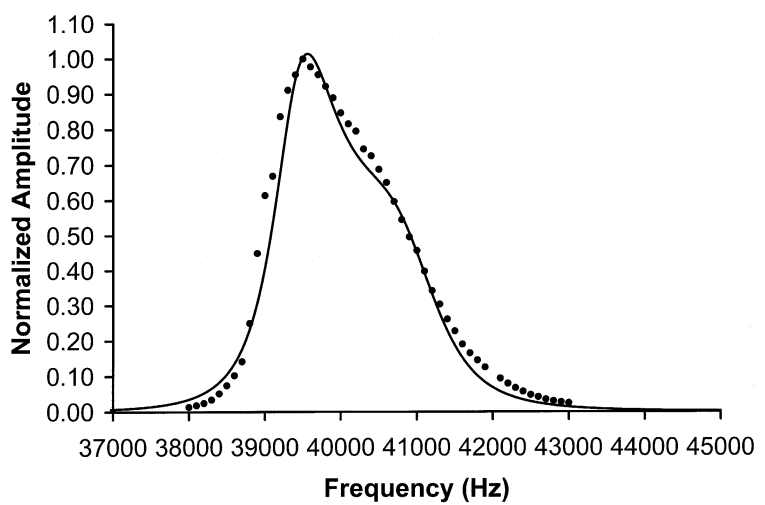

Fig. 4 - Amplitude response of a coupled pair of similar transducers with fitting process.

These best values which are the results of the software routine give the best approximation, according to the approximation criterion used for their selection.

In figure 4 data showing the amplitude response diagram for a pair of transducers of the same manufacturer, while in fig. 5 data showing the same diagram for a pair of transducers of different manufacturers. Dotted curves represent experimental data, while continuous curves represent theoretical data. 


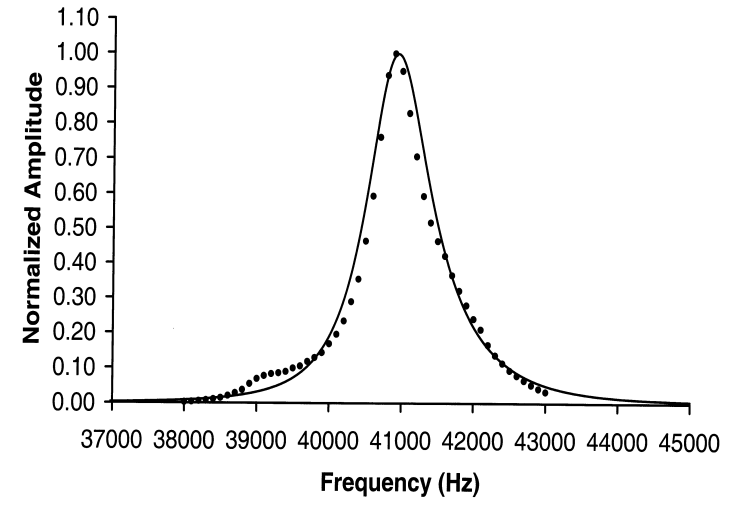

Fig. 5 - Amplitude response of a coupled pair of different transducers with fitting process.

Also for figure 4 the fitting process gave values of the transducers parameters: $\mathrm{f}_{0 \mathrm{~T}}=39718.75 \mathrm{~Hz}$, $\mathrm{BW}_{\mathrm{T}}=1046.875 \mathrm{~Hz}, \mathrm{f}_{0 \mathrm{R}}=40562.5 \mathrm{~Hz}, \mathrm{BW}_{\mathrm{R}}=$ $1484.375 \mathrm{~Hz}$, while for figure 5 the same process gave the following parameters: $\mathrm{f}_{0 \mathrm{~T}}=39390.63 \mathrm{~Hz}$, $\mathrm{BW}_{\mathrm{T}}=1046.875 \mathrm{~Hz}, \quad \mathrm{f}_{0 \mathrm{R}}=40937.5 \mathrm{~Hz}, \quad \mathrm{BW}_{\mathrm{R}}=$ $1593.75 \mathrm{~Hz}$.

As may be seen in these two typical cases presented here, we have a remarkable agreement between theoretical considerations and experimental measurements. Also we may now estimate very accurately the values of the main characteristic parameters of a set of transducers used in a specific measuring instrument. The experimental verification procedure has resulted to a situation where the value of the main parameters can be estimated while other, not well-known phenomena are clearly shown (i.e. the considerable difference between resonance frequencies or between bandwidth values).

It is obvious in the case of figure 5 that there is a significant difference between the frequencies $\mathrm{f}_{0 \mathrm{~T}}$ and $\mathrm{f}_{0 \mathrm{R}}$.

But regardless of it, the fitting process works properly and the approximation of experimental and theoretical curve appears to work effectively. The analysis of these data as a function of time provides information about the changes of the resonant frequency of each discrete transducer because of the ageing of the piezoelectric material.

\section{CONCLUSION}

An intelligent data acquisition system for the characterization and calibration of airborne ultrasonic transducers is presented. The proposed measuring system that is based on a DSP microcontroller is capable of making fast measurements of frequency and amplitude of the ultrasonic signal. Finally the proposed system used experimentally for the evaluation of different pairs of airborne ultrasound transducers operating in the range of $40 \mathrm{KHz}$. Such kind of results provides the proper information about the transducer parameters in steady state and transient response.

\section{REFERENCES}

[1]M.S. Obaidat, J.W. Ekis. An Automated system for characterizing ultrasonic transducers using pattern recognition. IEEE Trans. Instrum. and Measurement, Vol.40, N.5, Oct. 1991.

[2]A. Barwicz, J.L.Dion, R.Z. Morawski. Calibration of an electronic measuring system for ultrasonic analysis of solutions. IEEE Trans. Instrum. and Measurem. Vol.39, N.6, Dec. 1990.

[3]A.M. Sabatini. A digital signal-Processing technique for compensating ultrasonic sensors. IEEE Trans. Instrumentation and Measurement, Vol.44, N.4, Aug. 1995.

[4] Ch. Papageorgiou, T. Laopoulos. Accurate Time-of-Flight Measurement of Ultrasonic Signals for Displacement Monitoring Applications. IEEE. Instrumentation. and Measurement Technology Conference (IMTC'98), St. Paul, Minnesota, USA, May 1821, 1998, Volume I, Page(s) 154-159.

[5]Ch. Papageorgiou, C. Kosmatopoulos, Th. Laopoulos. Automated characterization and calibration of ultrasonic transducers. $9^{\text {th }}$ Mediterranean Electrotechnical Conference (MELECON '98), Tel-Aviv, Israel 1998, Volume II, Page(s): 1214 -1218.

[6]Ch. Papageorgiou, C. Kosmatopoulos, Th. Laopoulos. A method for remote measurements of velocity for vibration analysis. Proceedings of ICECS '99. The 6th IEEE International Conference on Electronics, Circuits and Systems, September 5-8, 1999, Pafos, Cyprus, Volume: III, Page(s): 1491 -1494.

[7] A.M. Sabatini. A Digital-Signal-Processing Technique for Ultrasonic Signal Modeling and Classification. IEEE Trans. Instrumentation and Measurement, Vol.50, N.1, February 2001

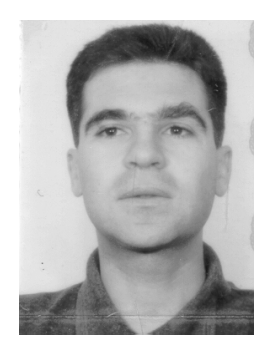

Chris Papageorgiou was born in Komotini, Greece. He received the B.S. degree in Physics and the M.Sc. degree in Electronics from the Aristotle University of Thessaloniki, in 1981 and 1984 respectively. $\mathrm{He}$ is currently finishing his Ph.D. studies at the same University. He has been involved in the research of ultrasonics since 1993, working in the areas of measurement systems with low frequency airborne ultrasonic transducers. Research interests include model analysis and characterization of these 
transducers, as well as digital signal processing of the transmitted-received ultrasonic waves for remote displacement, distance and vibration measurements, microcontroller applications, and electronics education.

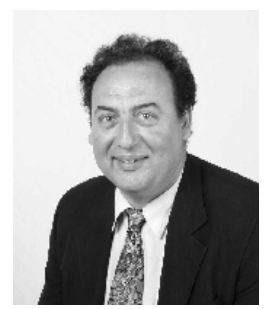

Theodore Laopoulos, was born in Thessaloniki, Greece, and studied Physics and Electronics at the Aristotle University of Thessaloniki, Greece. He is an Associate Professor at the Electronics Lab., Physics Department, Aristotle University of Thessaloniki, Greece. His research interests are in the fields of: Circuits and Systems for Low Frequency
Instrumentation, Automated Measurement Systems, Signal Processing for Measurement Systems, Sensor Interfacing and Control Electronics.

Dr. Laopoulos has published more than 75 papers in international scientific journals and conferences, is actively involved in research projects (National and European) in the fields of automated instrumentation and circuit design, and also in collaboration programs (research and education) with other European institutions. He is an active member of the IEEE Instrumentation and Measurement Society serving in the editorial board of the IEEE Instr. \& Meas. Transactions, and of the I\&M Magazine, and chairman of the Technical Committee "Education for I\&M". 